\title{
Locally conformally Kähler metrics obtained from pseudoconvex shells
}

\author{
Liviu Ornea ${ }^{1}$ and Misha Verbitsky 2
}

\begin{abstract}
A locally conformally Kähler (LCK) manifold is a complex manifold $M$ admitting a Kähler covering $\tilde{M}$, such that its monodromy acts on this covering by homotheties. A compact LCK manifold is called LCK with potential if its covering admits an authomorphic Kähler potential. It is known that in this case $\tilde{M}$ is an algebraic cone, that is, the set of all non-zero vectors in the total space of an anti-ample line bundle over a projective orbifold. We start with an algebraic cone $C$, and show that the set of Kähler metrics with potential which could arise from an LCK structure is in bijective correspondence with the set of pseudoconvex shells, that is, pseudoconvex hypersurfaces in $C$ meeting each orbit of the associated $\mathbb{R}^{>0}$-action exactly once. This is used to produce explicit LCK and Vaisman metrics on Hopf manifolds, generalizing earlier work by Gauduchon-Ornea and Kamishima-Ornea.
\end{abstract}

\section{Contents}

1 Introduction

1.1 Constructions of LCK metrics . . . . . . . . . . . . . 2

1.2 LCK manifolds . . . . . . . . . . . . . . . . 3

1.3 Survey of literature . . . . . . . . . . . . . . 4

2 Algebraic cones and LCK manifolds with potential 5 2.1 Algebraic cones . . . . . . . . . . . . 5

2.2 CR-geometry and Sasakian manifolds . . . . . . . . 6

\footnotetext{
${ }^{1}$ Partially supported by CNCS UEFISCDI, project number PN-II-ID-PCE-2011-30118.

${ }^{2}$ Partially supported by the RFBR grant 10-01-93113-NCNIL-a, Science Foundation of the SU-HSE award No. 10-09-0015 and AG Laboratory HSE, RF government grant, ag. 11.G34.31.0023
}

Keywords: Locally conformally Kähler manifold, Kähler potential, pseudoconvex, Sasakian manifold, Vaisman manifold, Hopf manifold.

2010 Mathematics Subject Classification: 53C55, 53C25. 
2.3 Pseudoconvex shells in algebraic cones . . . . . . . . . . 8

2.4 Vaisman metrics and pseudoconvex shells . . . . . . . . . . 10

2.5 Examples and erratum ................ 11

\section{Introduction}

\subsection{Constructions of LCK metrics}

Definition 1.1: A locally conformally Kähler (LCK) manifold is a complex manifold $M, \operatorname{dim}_{\mathbb{C}} M>1$, admitting a Kähler covering $(\tilde{M}, \tilde{\omega})$, with the deck transform group acting on $(\tilde{M}, \tilde{\omega})$ by holomorphic homotheties.

For equivalent definitions and examples, see [DO] or [OV6].

A (linear) Hopf manifold $H$ is a quotient of $\mathbb{C}^{n} \backslash 0$ by an action of $\mathbb{Z}$ generated by a linear map $A: \mathbb{C}^{n} \longrightarrow \mathbb{C}^{n}$, with all eigenvalues satisfying $\left|\alpha_{i}\right|<1$. It is easy to see that the Hopf manifold is diffeomorphic to $S^{1} \times$ $S^{2 n-1}$. Since $b_{1}(H)=1$ (an odd number), $H$ is non-Kähler. In fact, this manifold is probably the earliest example of a non-Kähler complex manifold known in mathematics.

However, any Hopf manifold is locally conformally Kähler. This observation originated in works of Izu Vaisman of 1970-ies; Vaisman defined and studied a strictly smaller class of manifolds, called by him "generalized Hopf". Now these manifolds are known as "Vaisman manifolds", because the name "generalized Hopf manifold" was already used by Brieskorn and van de Ven (see [BV]) for some products of homotopy spheres which do not bear Vaisman's structure. Besides, now it is known that not all Hopf manifolds belong to this smaller class.

These constructions are non-elementary. In fact, even the existence of locally conformally Kähler metrics on many Hopf surfaces is quite non-trivial. A clean but complicated argument was given in [GO], where such metrics were constructed in dimension 2 ; when the map $A$ is diagonal, the constructed metric is Vaisman. For non-diagonal $A$ the construction was much less explicit; in fact, the LCK metric on these Hopf surfaces was obtained only by deformation.

In the present paper we use the formalism of "locally conformally Kähler metrics with potential", that we built in previous papers, e.g. OV4, to obtain an explicit, computation-free and extremely simple construction of LCK metrics on manifolds which are obtained as $\mathbb{Z}$-quotients of algebraic varieties. 
This gives, among other things, the first explicit (i.e. not by deformations) construction of an LCK metric on a non-diagonal Hopf manifolds.

\subsection{LCK manifolds}

This section contains the definitions to be used in the paper. Unless otherwise stated, we only refer to compact, connected manifolds (although the definitions work also for noncompact manifolds).

Definition 1.2: A complex Hermitian manifold $(M, J, g)$ is locally conformally Kähler if its fundamental two-form $\omega:=g \circ J$ satisfies

$$
d \omega=\theta \wedge \omega, \quad d \theta=0
$$

here $\theta$ is called the Lee form. This definition is equivalent to the one given above.

A particular subclass of LCK manifolds is described in the following:

Definition 1.3: A Vaisman manifold is a LCK manifold whose Lee form is parallel with respect to the Levi Civita connection of $g$.

Compact Vaisman manifolds are equipped with a Riemannian submersion (a suspension in fact) to the circle with the fibers isometric to a Sasakian manifold $N$, see OV1] (and see [BG] for an introduction to Sasakian geometry). Their universal coverings are Riemannian cones $N \times \mathbb{R}^{>0}$ on which the deck transform group, isomorphic to $\mathbb{Z}$, acts by $(x, t) \mapsto(\varphi(x), q t)$, where $\varphi$ is a Sasakian automorphism of $N$ and $q \in \mathbb{N}$. The diagonal Hopf manifold is a typical example, see $\$ 1.3$. On the other hand, it is known, $[\mathrm{B}]$, that non-diagonal Hopf surfaces can never be Vaisman (although they are LCK, see [GO, OV4]).

A still wider subclass is the following:

Definition 1.4: An LCK manifold $M$ which admits a Kähler covering $(\tilde{M}, \tilde{\omega})$ with the Kähler form $\tilde{\omega}$ having a global, automorphic potential is called LCK manifold with potential. Here, by an automorphic potential we understand a function $\psi: \tilde{M} \longrightarrow \mathbb{R}$ satisfying $d d^{c} \psi=\tilde{\omega}$, with the monodromy of $\tilde{M}$ mapping $\psi$ to const $\cdot \psi$.

All Vaisman manifolds are LCK with potential (given by the squared norm of the Lee form with respect to the Kähler metric). As for Vaisman manifolds, the monodromy of LCK with potential manifolds is isomorphic 
to $\mathbb{Z}$. And hence this subclass is strict, as shown by the example of the LCK Inoue surfaces and of the LCK Oeljeklaus-Toma manifolds (see [OT]).

In this paper we shall be concerned with the linear Hopf manifolds. These are quotients of $\mathbb{C}^{n} \backslash\{0\}$ by the cyclic group generated by a linear operator with eigenvalues strictly smaller than 1 in absolute value. It is known that Hopf manifolds are LCK with potential, see [OV4], and Vaisman if the operator is diagonal.

\subsection{Survey of literature}

There are several papers where explicit constructions of LCK metric on diagonal Hopf manifolds appear.

The first one is $\mathrm{Va}$, where the metric (therein named after W. Boothby) $\frac{\sum d z_{i} \otimes d z_{i}}{\left|\sum z_{i} \bar{z}_{i}\right|^{2}}$ was considered on $\mathbb{C}^{n} \backslash\{0\} /\left\langle z_{i} \mapsto 2 z_{i}\right\rangle$.

More than twenty years took to pass from operators $A=\alpha \cdot I_{n}, \alpha \in \mathbb{C}$, to diagonal operators with complex non-equal eigenvalues. In GO, a LCK metric was constructed on diagonal Hopf surfaces $H_{\alpha, \beta}:=\mathbb{C}^{2} \backslash\{0\} /\langle(u, v) \mapsto$ $(\alpha u, \beta v)\rangle$. The construction is based on finding a Kähler potential on $\mathbb{C}^{2} \backslash$ $\{0\}$ in terms of $\alpha, \beta$, but the formula is only implicit. This procedure was generalized in $[\mathrm{B}$. The construction of Vaisman metrics in the present paper can also be considered as a generalization of [GO] to arbitrary dimensions.

In [KO] a construction was done for LCK metrics on $\mathbb{C}^{n} \backslash\{0\} /\left\langle z_{i} \mapsto\right.$ $\left.\alpha_{i} z_{i}\right\rangle$, starting from a deformation of the standard Sasakian structure of $S^{2 n-1}$ according to the $S^{1}$ action with weights $\alpha_{i}$ (cf. also GO, Section $3])$. The paper $[\mathrm{KO}$ ] also contains a very useful criterion to decide when a conformal class of LCK metrics on a complex manifold contains a Vaisman representative, in terms of the existence of a holomorphic complex flow which lifts to a non-trivial flow of homotheties of the Kähler covering.

A different construction on the same manifold, writing explicitly a Kähler potential on $\mathbb{C}^{n} \backslash\{0\}$, appeared in $[\mathrm{Ve}]$ and since then it was cited in almost all our subsequent papers. However, as observed by Matei Toma and Ryushi Goto, that metric is singular.

To correct this error we provide here a general construction of LCK metrics on Hopf manifolds. Our approach works for LCK manifolds with potential, giving a complete list of LCK metrics with potential in terms of a pseudoconvex shells in the covering (Definition 2.14).

For the time being, we don't know whether is it possible or not to write a formula for a potential for an LCK metric on a Hopf manifold; in the 
present paper, as well as in [GO], the potential is written as a solution of a certain differential equation.

\section{Algebraic cones and LCK manifolds with poten- tial}

\subsection{Algebraic cones}

Definition 2.1: A closed algebraic cone is an affine variety $C$ admitting a $\mathbb{C}^{*}$-action $\tau$ with a unique fixed point $x_{0}$ (called the origin), which satisfies the following.

1. $C$ is smooth outside of $x_{0}$.

2. $\tau$ acts on the Zariski tangent space $T_{x_{0}} \mathcal{C}$ diagonally, with all eigenvalues $\left|\alpha_{i}\right|<1$.

An open algebraic cone is a closed algebraic cone with the origin removed: $C \backslash\left\{x_{0}\right\}$.

Definition 2.2: Let $X$ be a projective orbifold, and let $L$ be an ample line bundle on $X$. Assume that the total space of $L$ is smooth outside of the zero divisor. The algebraic cone $\mathcal{C}(X, L)$ of $X, L$ is the total space of non-zero vectors in $L^{*}$. A cone structure on $\mathcal{C}(X, L)$ is the $\mathbb{C}^{*}$-action arising this way (by fiberwise multiplication).

In [OV3, Section 4], it was shown that any open algebraic cone $C$ is isomorphic to $\mathcal{C}(X, L)$, for appropriate $X$ and $L$. This was shown by the following argument. Given the algebraic cone $C$, one obtains $X$ as the quotient of $C$ by $\mathbb{C}^{*}$, and then the cone $C$ is naturally identified with the total space of a principal $\mathbb{C}^{*}$-bundle $L_{1}$. Ampleness of this bundle follows, because the corresponding closed algebraic cone $C_{c}$ is an affine variety, and algebraic functions on $C_{c}$ are identified with the section of the line bundle $L$ associated with $L_{1}$.

Definition 2.3: Let $\gamma$ be an automorphism of a closed algebraic cone. It is called a holomorphic contraction if for any compact subset $K \subset C$, and any open neighbourhood $U$ of the origin, there exists a number $N$ sufficiently big such that $\gamma^{N}(K) \subset U$. 
Definition 2.4: Let $C$ be a closed algebraic cone, and $\rho: \mathbb{R}^{>0} \longrightarrow \operatorname{Aut}(C)$ a $\mathbb{R}^{>0}$-action. We say that $\mathbb{R}^{>0}$ acts by holomorphic contractions, if $\rho(t)$ is a holomorphic contraction for all $t<1$.

Example 2.5: Let $C=\mathbb{C}^{n}=\mathcal{C}\left(\mathbb{C} P^{n-1}, \mathcal{O}(1)\right)$. Then any linear automorphism of $C$ with all eigenvalues $\left|\alpha_{i}\right|<1$ acts on $C$ by holomorphic contractions.

Example 2.6: Let $\rho:=\left.\tau\right|_{\mathbb{R}>0}$ be the action of $\mathbb{R}^{>0}$ on an algebraic cone provided by the cone structure, $\mathbb{R}^{>0} \subset \mathbb{C}^{*}$. Since $\rho$ acts on the tangent space $T_{c} C$ to the origin with eigenvalues smaller than 1 , it acts on $C$ by holomorphic contractions ([OV4, Theorem 3.3]).

Remark 2.7: As shown in OV4, Theorem 3.3], the quotient of an algebraic cone by a contraction is an LCK manifold with potential, and, conversely, any LCK manifold with potential is obtained by taking the quotient of an open algebraic cone by a holomorphic contraction. Such a contraction, being a priori a $\mathbb{Z}$-action, can be extended to a $\mathbb{R}^{>0}$-action by holomorphic contractions.

Further on, we use the following version of this result.

Theorem 2.8: Let $M$ be a locally conformally Kähler manifold with potential. Then $\tilde{M}$, as a complex manifold, is isomorphic to an open algebraic cone $C$, equipped with an action $\rho$ of $\mathbb{R}^{>0}$ by holomorphic contractions, and the quotient $\tilde{M} /\left\langle\rho\left(2^{n}\right)\right\rangle$ is isomorphic (as a complex manifold) to $M$.

Proof: In [OV5, Theorem 2.1], it is shown that $M$ can be deformed into a Vaisman manifold. From its proof it is apparent that this deformation preserves $\tilde{M}$ (in fact, only the $\mathbb{Z}$-action is deformed). Therefore, $\tilde{M}$ is a covering of a Vaisman manifold. Then, it is an algebraic cone, as follows from [OV2, Proposition 4.6].

\subsection{CR-geometry and Sasakian manifolds}

In this subsection, we introduce the Sasakian manifolds and some related notions of CR-geometry. We follow OV3]. 
Definition 2.9: A CR-structure (Cauchy-Riemann structure) on a manifold $M$ is a subbundle $H \subset T M \otimes \mathbb{C}$ of the complexified tangent bundle, which is closed under commutator: $[H, H] \subset H$ and satisfies $H \cap \bar{H}=0$.

A function $f: M \rightarrow \mathbb{C}$ is CR-holomorphic if $D_{V} f=0$ for any vector field $V \in \bar{H}$.

On a $\mathrm{CR}$ manifold $(M, H)$, the bundle $H \oplus \bar{H}$ is preserved by complex conjugation and hence it is obtained as a complexification of a real subbundle $H_{\mathbb{R}}$. Then $I_{H}:=-\sqrt{-1} \mathrm{I} \mathrm{d}_{\bar{H}}$ defines a complex structure on $H_{\mathbb{R}}$ and $H$ is its $\sqrt{-1}$-eigenspace of its extension to the complexification $H_{\mathbb{R}} \otimes \mathbb{C}$.

If $\operatorname{codim}_{T M} H_{\mathbb{R}}=1$, and the Frobenius tensor $L: H_{\mathbb{R}} \times H_{\mathbb{R}} \rightarrow T M / H_{\mathbb{R}}$, $L(X, Y)=[X, Y] \bmod H_{\mathbb{R}}$ is nondegenerate, then $(M, H)$ is a CR contact manifold and $H_{\mathbb{R}}$ is its contact structure (or distribution). In this context, $L$ is called the Levi form.

As $L$ vanishes on $H$ and $\bar{H}, L$ is $(1,1)$ with respect to $I_{H}$.

Definition 2.10: A contact CR-manifold $\left(M, H_{\mathbb{R}}, I_{H}\right)$ is called pseudoconvex if the Levi form is positive or negative, depending on the choice of orientation. If this form is also sign-definite, then $\left(M, H_{\mathbb{R}}, I_{H}\right)$ is called strictly pseudoconvex.

Definition 2.11: Let $S$ be a CR-manifold. A CR-holomorphic vector field $v \in T S$ is called transversal if it is transversal to the CR-distribution $H_{\mathbb{R}} \subset T S$.

Theorem 2.12: OV3, Theorem 1.2] Let $M$ be a compact pseudoconvex contact CR-manifold. Then the following conditions are equivalent.

(i) $M$ admits a Sasakian metric, compatible with the CR-structure.

(ii) $M$ admits a proper, transversal CR-holomorphic $S^{1}$-action.

(iii) $M$ admits a nowhere degenerate, transversal CR-holomorphic vector field.

Theorem 2.13: OV3, Theorem 1.3] Let $M$ be a compact, strictly pseudoconvex CR-manifold admitting a proper, transversal CR-holomorphic $S^{1}$ action. Then $M$ admits a unique (up to an automorphism) $S^{1}$-invariant CR-embedding into an algebraic cone $(\mathcal{C}, \tau)$. Moreover, a Sasakian metric 
on $M$ can be induced from a Kähler metric $\tilde{\omega}$ on this cone, which is automorphic in the following sense: for some constant $c>1$, one has $\tau(t)^{*} \tilde{\omega}=|t|^{c} \tilde{\omega}$.

\subsection{Pseudoconvex shells in algebraic cones}

Definition 2.14: Let $C$ be an algebraic cone, equipped with an action $\rho$ of $\mathbb{R}^{>0}$ by holomorphic contractions. A pseudoconvex shell in $C$ is a strictly pseudoconvex submanifold in $C$, intersecting each orbit of $\rho$ exactly once.

Remark 2.15: Please note that the action of $\rho$ may bear no relation to the cone action $\tau: \mathbb{C}^{*} \longrightarrow \operatorname{Aut}(C)$.

Theorem 2.16: Let $M=C /\langle\rho(q)\rangle$ where $(C, \tau)$ be an algebraic cone, equipped with the action $\rho$ of $\mathbb{R}^{>0}$ by holomorphic contractions and $q>1$. Let $\vec{r}$ be the infinitesimal generator of $\rho$ and let $S$ be a pseudoconvex shell in $C$. Then for each $\lambda \in \mathbb{R}$ there exists a unique function $\varphi_{\lambda}$ such that $\operatorname{Lie}_{\vec{r}} \varphi_{\lambda}=\lambda \varphi_{\lambda}$ and $\left.\varphi_{\lambda}\right|_{S}=1$. Moreover, such $\varphi_{\lambda}$ is plurisubharmonic for sufficiently big $\lambda \gg 0$. Conversely, any LCK manifold with potential admits a metric obtained this way.

Proof: For each $\rho$-orbit and each $\rho$-equivariant potential $\varphi$, one has:

$$
\rho(t) \cdot \varphi_{\lambda}=e^{t \lambda} \varphi_{\lambda}, \quad t \in \mathbb{R}^{>0} .
$$

Let $S$ be a pseudoconvex shell in $C$. Then $S \times \mathbb{R}^{>0} \stackrel{\sim}{\longrightarrow} C$, as orbits intersect the shell only once. Hence for any $s \in S$ and for any $t \in \mathbb{R}^{>0}$ we have:

$$
\varphi_{\lambda}(\rho(t) \cdot s)=e^{\lambda t}
$$

and this equation uniquely defines $\varphi_{\lambda}$. The problem is to prove that $d d^{c} \varphi_{\lambda}>$ 0 and this is not automatic for $\varphi_{\lambda}$, but it holds for some power of it.

Now let $B:=e^{\mathbb{R} \vec{r}} \cdot(T S \cap I(T S)) \subset T C$ be the subbundle obtained by translating $T S \cap I(T S)$ with all $e^{t \vec{r}}$. Then, by construction, $\left.d d^{c} \varphi_{\lambda}\right|_{B}$ is the Levi form of $B$ and hence it is positive definite.

It will now suffice to show that $\left.d d^{c} \varphi_{2 a \lambda}\right|_{S}=\left.d d^{c} \varphi_{\lambda}^{2 a}\right|_{S}>0$ for sufficiently big $a$. But

$$
d d^{c} \varphi_{\lambda}^{2 a}=\varphi_{\lambda}^{2 a-2}\left(2 a \varphi_{\lambda} \cdot d d^{c} \varphi_{\lambda}+2 a(2 a-1) d \varphi_{\lambda} \wedge d^{c} \varphi_{\lambda}\right)
$$


As the shell $S$ is compact, the result is implied by the following elementary linear algebra lemma:

Lemma 2.17: Let $h_{1}, h_{2}$ be pseudo-Hermitian forms on a complex vector space $V$ and let $W \subset V$ be a codimension 1 subspace. Assume that $\left.h_{1}\right|_{W}$ and $\left.h_{2}\right|_{V / W}$ are strictly positive (that is, positive definite), and $\left.h_{2}\right|_{W}=0$. Then there exists $u_{0} \in \mathbb{R}$, depending continuously on $h_{1}, h_{2}$, such that $h_{u}:=$ $h_{1}+u h_{2}$ is positive definite for any $u>u_{0}$.

The direct part of the Theorem now follows by applying Lemma 2.17 (whose proof we postpone) to $V=T M, W=B, h_{1}=\varphi_{\lambda} d d^{c} \varphi_{\lambda}, h_{2}=$ $d \varphi_{\lambda} \wedge d^{c} \varphi_{\lambda}$.

For the converse, let $\varphi_{\lambda}$ be any automorphic potential, thus satisfying $\left(\gamma^{k}\right)^{*}=e^{\lambda} \varphi_{\lambda}$, and let $\vec{r}$ be the holomorphic vector field which is the logarithm of the monodromy action. Let then $\rho(t)=e^{-t \lambda}\left(e^{t} \vec{r}\right)^{*}$ the corresponding endomorphism of $\mathcal{C}^{\infty}(M)$. Then $\rho(k+t)\left(\varphi_{\lambda}\right)=\rho(t) \varphi_{\lambda}$ and hence the orbit of $\rho$ through $\varphi$ is compact. We then average $\rho(t) \varphi$ on $\mathbb{R}$ and obtain a $\rho(t)$-invariant Kähler potential $\varphi_{\lambda 0}$. This $\varphi_{\lambda 0}$ is obtained from a pseudoconvex shell $S=\varphi_{\lambda 0}^{-1}(1)$ and from $\vec{r}$ as in the direct part of the Theorem.

Let us now give the proof of Lemma 2.17. For simplicity, we work in the real setting, and we consider $h_{1}, h_{2}$ as bilinear symmetric forms. Let $y \in V$ be a vector such that $h_{2}(y, y)=1$. Then any $x \in V$ can be written as $x=a y+z$, for some $z \in W$. This translates to:

$$
h_{u}(x, x)=u a^{2}+a^{2} h_{1}(y, y)+h_{1}(z, z)+2 a h_{1}(z, y)
$$

which we view as a polynomial in $a$. This one is positive definite for all $a$ if and only if

$$
\left(h_{1}(z, y)\right)^{2}-\left(u+h_{1}(y, y)\right) \cdot h_{1}(z, z)<0 .
$$

Choose $y^{\prime} \in W$ such that $h_{1}\left(z, y^{\prime}\right)=h_{1}(z, y)$ for all $z \in W$ and let $u>u_{0}:=$ $h_{1}\left(y^{\prime}, y^{\prime}\right)-h_{1}(y, y)$. Then (2.2) becomes

$$
\left(h_{1}\left(z, y^{\prime}\right)\right)^{2}-h_{1}\left(y^{\prime}, y^{\prime}\right) h_{1}(z, z)<0,
$$

which is satisfied by Cauchy-Buniakovski-Schwarz inequality, as $h_{1}$ is positive definite on $W$.

Example 2.18: Let $A$ be a linear operator on $\mathbb{C}^{n}$ with eigenvalues of absolute values strictly smaller than 1 . Let $C=\mathbb{C}^{n} \backslash\{0\}$ and let $\rho(t)=e^{t \log A}$. 
Take a sphere $S=S^{2 n-1} \subset \mathbb{C}^{n}$; it is easy to see that $S$ is a pseudoconvex shell. Applying Theorem 2.16, we obtain an automorphic potential $\varphi$ on $\mathbb{C}^{n} \backslash 0$, giving an LCK metric on $M=\left(\mathbb{C}^{n} \backslash 0\right) /\langle A\rangle$.

Remark 2.19: When $n=2$ and $A$ is diagonal, the same potential was obtained in [GO].

In particular, we recover the result proven in [GO, $\mathrm{KO}$, OV4] that all Hopf manifolds $\left(\mathbb{C}^{n} \backslash\{0\}\right) /\langle A\rangle$ are $L C K$.

\subsection{Vaisman metrics and pseudoconvex shells}

Remark 2.20: Vaisman manifolds are LCK with potential and hence they have canonical pseudoconvex shells (levels of the potential).

Definition 2.21: Let $(M, I, g)$ be a Vaisman manifold, let $(C, \rho)$ be the associated algebraic cone, equipped with the action $\rho$ of $\mathbb{R}^{>0}$ by holomorphic contractions, and let $S$ be its pseudoconvex shell. The Reeb field of $M$ is the CR-holomorphic vector field $I \theta^{\sharp} \in T S$ obtained from $\rho$ by complex conjugation.

Remark 2.22: For Vaisman manifolds, the Reeb field is always transversal ( OV3]).

Proposition 2.23: Let $v \in T S$ be a CR-holomorphic vector field on $S$, where $S$ is a pseudoconvex shell in an algebraic cone $C$. Then $v$ can be uniquely extended to a holomorphic vector field on the whole of $C$. 1

Proof: Let $\mathcal{O}_{S}$, respectively $\mathcal{O}_{S}$ 。 be the ring of CR-holomorphic functions on $S$, respectively on the interior $S^{\circ}$ of the shell $S$. By the solution of the Neumann problem, $L^{2}\left(\mathcal{O}_{S}\right)=L^{2}\left(\mathcal{O}_{S^{\circ}}\right)$ and hence, if we restrict to bounded functions, $\mathcal{O}_{S^{\circ}}=\mathcal{O}_{S}$ as rings. As vector fields on $S$ and $S^{\circ}$ are derivations of the above rings, the result follows.

Theorem 2.24: Let $(M, I, g)$ be an LCK manifold obtained (as in Theorem 2.16) from an algebraic cone $C$ and a pseudoconvex shell $S$, and let $\gamma: \mathbb{Z} \longrightarrow \operatorname{Aut}(C)$ be the deck transform map. Then the Hermitian manifold $(M, I, g)$ is conformally equivalent to a Vaisman one if and only if $S$ admits a transversal

\footnotetext{
${ }^{1}$ This is called the holomorphic extension of $v$.
} 
CR-holomorphic vector field $\xi$, such that its holomorphic extension to $C$ is $\gamma(1)$-invariant and $\exp (-I \xi) \cdot \gamma(1)$ preserves $S$.

Proof: Suppose $(M, I, g)$ is Vaisman. Then $\xi=I \theta^{\sharp}$ is an isometry of the LCK metric. The Kähler metric on $C$ is $d d^{c} \varphi$, with $\varphi$ given by the equation

$$
e^{t \theta^{\sharp}}(S)=\varphi^{-1}(t),
$$

where $S$ is the level set of $\varphi$. Then $S$ is Sasakian and $I \theta^{\sharp}$ is the Reeb field of the underlying contact structure, hence it is transversal by definition. By construction, $M=C /\langle\gamma(1)\rangle$, and the extension of $\xi$ to $C$ is the lift to $C$ and is $\gamma(1)$ invariant by definition. We have checked all the conditions of Theorem 2.24, except the last one: $\exp (-I \xi) \cdot \gamma(1)(S)=S$.

The Lee field $-I \xi=\theta^{\sharp}$ acts by homotheties on the potential $\varphi$, hence $\mathrm{Lie}_{\exp (-I \xi)} \varphi=c \varphi$ for some contant $c$. The monodromy map $\gamma(1)$ also acts by homotheties on $\varphi$ : $\operatorname{Lie}_{\rho(1)} \varphi=c^{\prime} \varphi$ for another constant $c^{\prime}$. What we have to do is to homothetically modify the initial metric $g$ such that $c$ becomes $1 / c^{\prime}$; in this case $\exp (-I \xi) \cdot \gamma(1)$ will preserve the potential $\varphi$ and hence will preserve $S$.

Conversely, the vector field $\xi$ is tangent to $S$ and its flow $\exp (t \xi)$ acts by contractions, hence defining a metric on the cone $C$ over $S$. As $\gamma(1)$ maps a shell $S_{\lambda}$ to $S_{\text {const. } \lambda}$, if follows that $\gamma(1)$ acts by homotheties on $C$. This means that the complex flow generated on $M=C /\langle\gamma(1)\rangle$ by $\xi$ and $I \xi$ lifts to a flow of non-trivial homotheties on the cone. By [KO] this ensures the existence of a Vaisman metric in the conformal class of $g$.

\subsection{Examples and erratum}

As an application, we add an erratum to several papers where we have given a wrong formula for an LCK-metric on diagonal Hopf manifolds (e.g. [Ve], [O]), and give a general and almost explicit construction of a Vaisman metric on any diagonal Hopf manifold. This constructions originates in the ones in $\mathrm{GO}, \mathrm{B}, \mathrm{KO}$, but unifies them and presents them in a much more synthetic and transparent way.

We apply Theorem 2.24. The data to start with are the cone $C$, the shell $S$, the vector field $\xi$ and the monodromy $\gamma$.

Let $A=\operatorname{diag}\left(\alpha_{1}, \cdots, \alpha_{n}\right)$, with $0<\left|\alpha_{1}\right| \leqslant\left|\alpha_{2}\right| \leqslant \cdots \leqslant\left|\alpha_{n}\right|<1$. Denote by $A_{|\cdot|}$ the matrix $\operatorname{diag}\left(\left|\alpha_{1}\right|, \cdots,\left|\alpha_{n}\right|\right)$ (in the same basis). 
Let $C$ be $\mathbb{C}^{n} \backslash\{0\}$. As a shell $S$, we take the sphere $S^{2 n-1}$, but one can take for $S$ the boundary of any strictly pseudoconvex body containing 0 and satisfying $A_{|\cdot|} A^{-1}(S)=S$.

The transversal CR-holomorphic vector field $\xi$ is

$$
\xi=\sqrt{-1} \operatorname{Re} \log A=\sqrt{-1} \log \operatorname{diag}\left(\left|\alpha_{1}\right|, \ldots,\left|\alpha_{n}\right|\right)
$$

and the monodromy map $\gamma$ is given by $\gamma(z)=A \cdot z, z \in C$.

To apply Theorem 2.24 we need to verify that $\xi$ is $\gamma(1)$-invariant and $\exp (-I \xi) \cdot \gamma(1)$ preserves $S$. An easy computation shows that $\exp (-I \xi) \cdot \gamma(1)$ acts as $A_{|\cdot|} A^{-1}=\operatorname{diag}\left(\frac{\alpha_{1}}{\left|\alpha_{1}\right|}, \cdots, \frac{\alpha_{n}}{\left|\alpha_{n}\right|}\right)$ and thus it preserves the norm of vectors, hence preserving the spheres. Finally, as the action of $\gamma(1)$ is linear, the $\gamma(1)$-invariance of $\xi$ amounts to $A \cdot \xi_{z}=\xi_{A \cdot z}$ which is immediate from (2.3).

This gives an explicit construction of Vaisman structure on diagonal Hopf manifolds. On the other hand, Theorem 2.16 provides a rather explicit construction of LCK metrics on non-diagonal Hopf manifolds, avoiding the argument by deformations in OV4.

Acknowledgments: We thank Matei Toma and Ryushi Goto for drawing our attention to the wrong formula which originated this paper, and to Max Planck Institute for Mathematics (Bonn), where this paper was finished, for the excellent research environment.

\section{References}

[B] F.A. Belgun, On the metric structure of non-Kähler complex surfaces, Math. Ann. 317 (2000), 1-40.

[BG] C.P. Boyer, K. Galicki, Sasakian geometry. Oxford Mathematical Monographs. Oxford University Press, Oxford, 2008.

[BV] E. Brieskorn, A. van de Ven, Some complex structures on products of homotopy spheres, Topology 7 (1968) 389-393.

[DO] S. Dragomir and L. Ornea, Locally conformal Kähler geometry, Progress in Math. 155, Birkhäuser, Boston, Basel, 1998.

[GO] P. Gauduchon and L. Ornea, Locally conformally Kähler metrics on Hopf surfaces, Ann. Inst. Fourier (Grenoble) 48 (1998), 1107-1127.

[KO] Y. Kamishima and L. Ornea, Geometric flow on compact locally conformally Kähler manifolds, Tohoku Math. J. 57 (2005), 201-221.

[OT] K. Oeljeklaus and M. Toma, Non-Kähler compact complex manifolds associated to number fields, Ann. Inst. Fourier (Grenoble) 55 (2005), no. 1, 161-171. 
[O] L. Ornea, Locally conformally Kaehler manifolds. A selection of results. Lecture Notes of Seminario Interdisciplinare di Matematica, 4 (2005), 121-152.

[OV1] L. Ornea and M. Verbitsky, Structure theorem for compact Vaisman manifolds, Math. Res. Lett., 10 (2003), 799-805.

[OV2] L. Ornea and M. Verbitsky, An immersion theorem for compact Vaisman manifolds, Math. Ann. 332 (2005), no. 1, 121-143, see also math.AG/0306077

[OV3] L. Ornea and M. Verbitsky, Sasakian structures on CR-manifolds, math.DG/0606136, Geom. Dedicata 125 (2007), 159-173.

[OV4] L. Ornea and M. Verbitsky, Locally conformally Kähler manifolds with potential, Mathematische Annalen, 248 (1) (2010), 25-33, math.AG/0407231.

[OV5] L. Ornea and M. Verbitsky, Topology of locally conformally Kahler manifolds with potential, arXiv:0904.3362 Int. Math. Res. Not., 4, 717-726 (2010).

[OV6] L. Ornea and M. Verbitsky, A report on locally conformally Kähler manifolds, Contemporary Mathematics 542, 135-150, 2011. arXiv:1002.3473

[Va] I. Vaisman, On locally conformal almost Khler manifolds Israel J. Math. 24 (1976), 338-351.

[Ve] M. Verbitsky, Theorems on the vanishing of cohomology for locally conformally hyperKähler manifolds, Proc. Steklov Inst. Math. 246 (2004) 54-78. arXiv:math/0302219

LIVIU ORNEA

University of Bucharest, Faculty of Mathematics, 14 ACAdEMiei Str., 70109 Bucharest, Romania. and

Institute of Mathematics "Simion Stoilow" of the Romanian Academy, 21, Calea Grivitei Street 010702-Bucharest, Romania

Liviu.Ornea@imar.ro, lornea@gta.math.unibuc.ro

Misha Verbitsky

Laboratory of Algebraic Geometry, National Research University HSE,

7 Vavilova Str. Moscow, Russia, 117312

verbit@mccme.ru, verbit@verbit.ru 